\title{
BRAIN INJURY IN THE NEWBORN: NOT A MATTER OF GRAY OR WHITE
}

\section{S.P. Miller}

University of British Columbia, Vancouver, BC, Canada

The ability to image the newborn brain early in life reveals typical patterns of brain injury following insults at vulnerable time periods. Brain imaging studies have also provided valuable new information regarding the effects of injury on subsequent brain development. We now know that injury at term results in a predilection for gray matter injury while injury in the premature brain results predominantly in a pattern of white matter damage. These injuries affect how the brain subsequently matures with changes in connectivity that have profound functional consequences. This presentation will focus on these patterns of injury that are so critically determined by age at insult. In addition, this presentation will address how brain injury in the preterm newborn impacts gray matter development and how certain populations of term newborns are exceptionally sensitive to white matter injury. 